\title{
ENVELHECIMENTO PROFISSIONAL: PERCEPÇÕES E PRÁTICAS DE GESTÃO DA IDADE
}

PROFESSIONAL AGING: PERCEPTIONS AND PRACTICES OF AGE MANAGEMENT

ENVEJECIMIENTO PROFESIONAL: PERCEPCIONES Y PRÁCTICAS DE LA GESTIÓN DE LA EDAD

\author{
VANESSA MARTINES CEPELLOS \\ Doutora \\ Fundação Armando Álvares Penteado - Brasil \\ vmcepellos@gmail.com \\ MARIA JOSÉ TONELLI \\ Doutora \\ Fundação Getúlio Vargas - Brasil \\ maria.jose.tonelli@fgv.br \\ Submetido em: 06/02/2017 \\ Aprovado em: 03/04/2017 \\ Doi: alcance.v24n1.p4-21
}

\begin{abstract}
RESUMO
O envelhecimento é um dos fenômenos mais significativos do século XXI. Apesar dos dados demográficos enfatizarem a relevância do envelhecimento em nosso país, a literatura acadêmica ainda não aborda o tópico de forma abrangente. Assim, este estudo investiga quais as percepções de gestores de Recursos Humanos de empresas instaladas no Brasil a respeito dos profissionais com mais de 50 anos e quais são as práticas de gestão de idade adotadas por suas empresas. Os dados foram coletados junto a 138 gestores de RH por meio do preenchimento de questionário on-line e analisados estatisticamente. Os resultados permitiram identificar que, apesar do rápido envelhecimento populacional brasileiro, as empresas estudadas não estão preparadas para 0 envelhecimento da força de trabalho. As práticas de gestão da idade se mostram pouco adotadas pelas empresas estudadas, ainda que a percepção dos gestores acerca dos profissionais mais velhos seja relativamente positiva. $\mathrm{O}$ artigo contribui para a área de RH na medida em que apresenta a discussão sobre as percepções de gestores e práticas de gestão de idade, tópicos que, embora abordados internacionalmente, ainda não tinham sido explorados na literatura acadêmica nacional. 0 estudo pretende despertar 0 interesse de profissionais da área para uma maior reflexão sobre as condições de trabalho de profissionais mais velhos.
\end{abstract}

Palavras-chave: Envelhecimento. Práticas de Gestão. Percepção.

\section{ABSTRACT}

Aging is one of the most significant phenomena of the twenty-first century. Although the demographic data emphasize the importance of aging in our country, the academic literature still has not addressed the topic in a comprehensive way. This study investigates the perceptions of Human Resources managers of companies in Brazil, concerning professionals aged over 50 years, and the age management practices adopted by their companies. Data were collected from 138 HR managers who were asked to fill out online questionnaire. Their responses were then statistically analyzed. The results showed that despite the rapid aging of the Brazilian population, the companies studied are not prepared for an aging workforce. Age management practices have been little adopted by the companies studied, although managers' perceptions of older professionals are relatively positive. This article contributes to the area of HR by presenting a discussion of managers' perceptions age management practices. These are topics that, although addressed internationally, have not yet been explored by 
national Brazilian academic authors. The study also aims to attract the interest of professionals in the area, to promote further reflection on the working conditions of conditions of older professionals.

Keywords: Aging. Management practices of age. Perception.

\section{RESUMEN}

El envejecimiento es uno de los fenómenos más significativos del siglo XXI. Pese a que los datos demográficos enfatizan la relevancia del envejecimiento en nuestro país, la literatura académica aún no aborda este tópico de forma abarcadora. Por ese motivo, este estudio investiga cuáles son las percepciones de los gestores de Recursos Humanos de empresas instaladas en Brasil con respecto a los profesionales de más de 50 años y cuáles son las prácticas de gestión de edad adoptadas por sus empresas. Los datos fueron recogidos con 138 gestores de RRHH por medio de un cuestionario rellenado on-line y analizados estadísticamente. Los resultados permitieron identificar que, a pesar del rápido envejecimiento poblacional brasileño, las empresas estudiadas no están preparadas para el envejecimiento de la fuerza de trabajo. Las prácticas de gestión de la edad se muestran poco adoptadas por las empresas estudiadas, aunque la percepción de los gestores acerca de los profesionales de más edad sea relativamente positiva. El artículo contribuye con el área de RRHH en la medida en que presenta la discusión sobre las percepciones de gestores y prácticas de gestión de edad, tópicos que, aunque abordados internacionalmente, todavía no habían sido explotados en la literatura académica nacional. El estudio pretende despertar el interés de profesionales del área hacia una mayor reflexión sobre las condiciones de trabajo de profesionales mayores.

Palabras clave: Envejecimiento. Prácticas de gestión. Percepción.

\section{INTRODUÇÃO}

O envelhecimento populacional é um dos mais significativos fenômenos do século XXI e apresenta importantes implicações para todos os domínios da sociedade (FUNDO DE POPULAÇÃO DAS NAÇÕES UNIDAS, 2012). Iniciado em países desenvolvidos e industrializados, atualmente 0 envelhecimento também se apresenta em economias em desenvolvimento (GAVRILOV; HEUVELINE, 2003), modificando a imagem de um mundo com alta proporção de crianças e baixas proporções de idosos (PHILLIPS; SIU, 2012).

No Brasil, o processo de envelhecimento populacional está acontecendo de forma acelerada, implicando diretamente no envelhecimento da força de trabalho. Segundo estimativas, em 2040 os empregos estarão concentrados na população com idade acima dos 45 anos, um cenário que irá exigir iniciativas de gestão específicas às necessidades destes profissionais (INSTITUTO DE PESQUISA ECONÔMICA APLICADA [IPEA], 2006). Para se ter uma ideia, projeções do IBGE (2013) apontam que o aumento na participação relativa do grupo de idosos passará de 13,8\%, em 2020, para 33,7\%, em 2060.

Apesar da relevância do tópico, os estudos sobre envelhecimento ainda se mostram incipientes na literatura acadêmica. Na literatura nacional, os estudos exploram o fenômeno sob uma perspectiva teórica e se mostram pouco analíticos em termos de como os profissionais mais velhos são percebidos pelos gestores de $\mathrm{RH}$. Carvalho (2009), por exemplo, realizou um trabalho exploratório, que partiu de um levantamento bibliográfico sobre a contratação de idosos. Fontoura e Piccinini (2012) analisaram as publicações - principalmente internacionais que versam sobre o papel das ações de Gestão de Pessoas como mediadoras do processo de envelhecimento. Locatelli e Fontoura (2013) também se ativeram a realizar um levantamento das publicações a respeito do tema na área da Administração. Loth e Silveira (2014) buscaram compreender quais os estereótipos percebidos por envelhecentes em relação ao preconceito de idade e Silva et al. (2014) se dedicaram a analisar a gestão de pessoas de organizações que atuam no Brasil no que concerne a questões geracionais. Apesar de Locatelli e Fontoura (2013) concluírem que o número de pesquisas sobre o envelhecimento tem aumentado discretamente, não foram identificados trabalhos que abordassem, de forma ampla e empírica, o fenômeno no Brasil. É neste sentido que o estudo visa contribuir para o enriquecimento da discussão acerca do tópico.

Na literatura internacional, um dos tópicos amplamente discutidos é o ageism, ou seja, a discriminação de adultos mais velhos (BUTLER, 1989). O ageism tem sido estudado a partir da perspectiva da percepção de gestores de RH (BROOKE; TAYLOR, 2005; HASSEL; PERREWE, 1995; HENKENS, 2005; POSTHUMA; 
CAMPION, 2009; ROSCIGNO et al, 2007; TAYLOR; WALKER, 1998) ou, ainda, a partir da própria literatura existente sobre o tema (VASCONCELOS, 2015). Autores também se dedicaram a desenvolver um modelo de mudança social sustentável tendo em vista o envelhecimento da força de trabalho (ANGELONI; BORGOLOVI, 2016) e a influência de competências e satisfação com a carreira dos trabalhadores mais velhos (HENNEKAM, 2015). São discutidas também as práticas de gestão da idade que devem ser adotadas nas organizações, a fim de combaterem as barreiras etárias (MALATEST; ASSOCIADOS, 2003; NAEGELE; WALKER, 2006, ARMSTRONG-STASSEN, 2008). Estudos internacionais constataram que essas práticas, além de se mostrarem complexas e contraditórias (HENNEKAM; HERRBACH, 2015), não têm sido adotadas pelos gestores de RH com a frequência em que deveriam, dificultando a inserção e a manutenção destes profissionais nas organizações (HEDGE; BORMAN; LAMMLEIN, 2006; ARMSTRONG-STASSEN, 2008; ZNIDARSIC; DIMOVSKI, 2009).

Ainda que diversos estudos internacionais abordem o tópico do envelhecimento nas organizações, 0 conhecimento sobre as percepções de gestores de $\mathrm{RH}$ a respeito dos profissionais mais velhos e a adoção de práticas de gestão de idade em empresas que exercem atividades no Brasil são bastante reduzidos. Por esta razão, considerou-se oportuno verificar quais são as percepções positivas e negativas de gestores de $\mathrm{RH}$ a respeito dos profissionais com mais de 50 anos de idade e quais são as práticas de gestão da idade adotadas pelas empresas pesquisadas.

O artigo apresenta na primeira seção a fundamentação teórica; na segunda seção, os procedimentos metodológicos deste trabalho; seguidos da discussão dos resultados e as considerações finais.

\section{FUNDAMENTAÇÃO TEÓRICA}

No referencial teórico deste trabalho serão abordados os principais tópicos que serviram de base para a realização deste estudo: as percepções a respeito de profissionais mais velhos e as práticas de gestão da idade.

\subsection{Percepções sobre profissionais mais velhos}

Ainda que não exista uma definição clara acerca de quem pode ser considerado um profissional mais velho (LAZAZZARA; BOMBELLI, 2011), os autores Kooij et al. (2008) apontam que o termo profissional mais velho é utilizado para se referir a profissionais de 40 até 75 anos, dependendo do contexto de estudo. A dificuldade de estabelecer uma definição do profissional mais velho considerando somente a idade cronológica parece ser insuficiente, porque esses limites não se apresentam apenas em função da idade, mas também em função da cultura (HENRARD, 1996). Ainda assim, é possível verificar a discriminação de adultos mais velhos por conta de sua idade, fenômeno denominado ageism. $O$ termo ageism foi utilizado pela primeira vez pelo gerontologista Robert N. Butler, em 1968, para descrever a discriminação etária (PALMORE, 1999). Para Butler (2010), o ageism se manifesta por meio de diversos fenômenos, seja individual ou institucionalmente, por meio de estereótipos, mitos e práticas discriminatórias no emprego. As percepções acerca do indivíduo mais velho também podem refletir a existência da discriminação etária.

Alguns estudos internacionais abordaram o tópico e identificaram as principais percepções, tanto positivas quanto negativas, de gestores com relação aos profissionais mais velhos (BROOKE; TAYLOR, 2005; HASSEL; PERREWE, 1995; HENKENS, 2005; POSTHUMA; CAMPION, 2009; ROSCIGNO et al., 2007; TAYLOR; WALKER, 1998).

Segundo Hassel e Perrewe (1995), os funcionários, quando questionados a respeito de crenças e estereótipos sobre profissionais mais velhos, foram neutros ou não quiseram se comprometer com as respostas. Todavia, os autores identificaram alguns pontos negativos associados ao envelhecimento: maior dependência, preferência por trabalhos com menos desafios e o fato de não se mostrarem amigáveis nem extrovertidos no trabalho.

Na pesquisa de Taylor e Walker (1998), os gestores respondentes tendiam a pensar na incapacitação do profissional mais velho ao trabalho físico pesado, na dificuldade de serem treinados e no desinteresse em atividades de aprendizagem. Maior lentidão no trabalho, desorganização e problemas de relacionamento foram percepções negativas associadas aos mais velhos identificadas na pesquisa de Roscigno et al. (2007). Pontos de tensão entre profissionais de faixas etárias diferentes também ficaram evidentes na pesquisa de Brooke e Taylor (2005). Para esses autores, os profissionais mais velhos causam tensão no ambiente de trabalho quando respondem a profissionais mais jovens que ocupam cargos de supervisão. Para Henkens (2005), as percepções

Revista Alcance - Eletrônica - vol. 24 - n. 1 - jan./mar. 2017 
negativas estariam, ainda, associadas à adaptabilidade às novas tecnologias e às mudanças nas empresas.

Contudo, os gestores não possuem somente percepções negativas a respeito dos profissionais mais velhos. Taylor e Walker (1998) averiguaram que atributos como maior confiabilidade, produtividade, criatividade e menor propensão de sofrerem acidentes estavam associados aos profissionais com mais idade.

Dennis e Thomas (2007) constataram que, muitas vezes, os mais velhos também são valorizados por suas experiências, conhecimentos, hábitos de trabalho. Maior compromisso com a qualidade, lealdade, pontualidade, capacidade de manter a calma em momentos de crise e o respeito pela autoridade foram algumas das qualidades percebidas em relação ao profissional maduro. Além disso, eles foram avaliados pelo seu desempenho e competências básicas necessárias para o trabalho. Menores índices de turnover voluntário e absenteísmo também foram identificados como qualidades associadas aos hábitos de trabalho dos profissionais mais velhos (HASSEL; PERREWE, 1995; ROSCIGNO et al., 2007).

Em síntese, são diversas as percepções de gestores acerca do profissional mais velho identificadas em estudos internacionais. No Brasil, no entanto, não há estudos a respeito do tópico. Sendo assim, o levantamento das principais percepções abordadas foi essencial para a construção do questionário desta pesquisa, uma vez que um dos objetivos deste estudo é, justamente, identificar quais são as percepções positivas e negativas de gestores de RH a respeito dos profissionais mais velhos. Além deste objetivo, este estudo também pretende identificar quais são as práticas de gestão da idade adotadas pelas empresas pesquisadas. Por esta razão, é fundamental compreender 0 que vem a ser essas práticas de gestão de idade e o que os estudos dizem sobre elas.

\subsection{Práticas de Gestão da idade}

As práticas de gestão da idade são condutas que visam minimizar os efeitos do envelhecimento do profissional nas organizações e minimizar as barreiras etárias. Algumas tentativas de promover práticas direcionadas à manutenção e à retenção dos profissionais mais velhos foram apresentadas nos últimos anos. Uma delas se refere a um relatório idealizado por Malatest e Associados (2003), apresentado no Canadá e a outra se refere a um guia de boas práticas coordenado pela Fundação Europeia, de autoria de Naegele e Walker (2006), em que são descritos os principais fatores que envolvem as práticas na gestão de uma força de trabalho madura. Essas práticas estão ligadas ao processo de recrutamento, formação, desenvolvimento e promoção de profissionais, como também ao trabalho flexível, ergonomia e mudança de atitudes nas organizações (MALATEST; ASSOCIADOS, 2003; NAEGELE; WALKER, 2006).

Iniciativas de adoção de práticas de gestão voltadas aos profissionais mais velhos foram apresentadas e discutidas em estudos acadêmicos internacionais (MANPOWER, 2007; ARMSTRONG-STASSEN, 2008; ZNIDARSIC; DIMOVSKI, 2009; LAZAZZARA; BOMBELI, 2011). Em estudo realizado por Armstrong-Stassen (2008), foi identificado que, na visão da maioria dos profissionais mais velhos, estas práticas não são prioritárias para as empresas e cerca de $50 \%$ alegaram falta de interesse dos empregadores na busca de práticas eficazes. Znidarsic e Dimovski (2009) investigaram a visão dos gestores de RH da Eslovênia sobre as práticas de gestão da idade adotadas nas empresas e apuraram que elas ainda estavam em sua fase inicial, uma vez que não existiam evidências de estratégias direcionadas para a manutenção, formação e recrutamento de trabalhadores mais velhos.

Na direção oposta ao que pretendem as práticas de gestão de idade, algumas condutas reforçam o ageism na esfera organizacional. Estudos mostram que a idade pode se apresentar como fator importante durante o processo de recrutamento e seleção, inviabilizando a inserção de profissionais nas empresas (LORETTO; WHITE, 2006; PERES, 2003; TAYLOR; WALKER, 1998). No entanto, Naegele e Walker (2006) lembram que a organização deve promover acesso igual para qualquer candidato e não o discriminar, direta ou indiretamente, por conta da idade. Na mesma direção, o relatório de Malatest e Associados (2003) alerta para a necessidade de novos métodos de recrutamento que devem ser apropriados para os mais velhos.

No campo da formação e da aprendizagem, as práticas devem garantir que os mais velhos não sejam desprivilegiados no treinamento e no desenvolvimento de carreira (NAEGELE; WALKER, 2006) e práticas de retenção devem preservar as experiências acumuladas dos profissionais mais velhos (MALATEST; ASSOCIADOS, 2003).

Nota-se, portanto, a partir do levantamento de estudos a respeito das práticas de gestão de idade, a 
necessidade de aprofundar o tópico e de averiguar como essas práticas têm sido desenvolvidas pelas empresas no Brasil. A partir das práticas apresentadas ao longo do referencial teórico, foi possível elaborar o questionário deste estudo, como será visto na seção a seguir.

\section{METODOLOGIA}

A fim de atingir os objetivos propostos neste trabalho, que são investigar quais as percepções de gestores de RH de empresas instaladas no Brasil a respeito dos profissionais com mais de 50 anos e quais são as práticas de gestão de idade adotadas por suas empresas, foi realizada uma pesquisa quantitativa com 138 gestores de $\mathrm{RH}$ acessados a partir da base dados de uma das maiores consultorias e auditorias do país - a PricewaterhouseCoopers (PwC).

Apesar de não haver consenso na definição de profissional mais velho por gestores e empregadores (CLAES; HEYMANS, 2008), no presente estudo adotou-se a definição de Fontoura e Piccinini (2012), qual seja, de que o profissional mais velho é aquele com 50 anos ou mais, uma vez que esta idade pode ser considerada um ponto de referência em que os marcadores do processo de envelhecimento tornam-se mais visíveis para a maioria das pessoas.

Adotou-se como instrumento de coleta de dados um questionário elaborado pelos autores deste artigo com base em estudos internacionais. $O$ instrumento de pesquisa foi submetido à apreciação de especialistas da área de estatística e de administração para sua validação. Inicialmente, foi realizado um pré-teste com 70 respondentes, que permitiu seu aperfeiçoamento para a pesquisa final. Na sequência, o questionário foi enviado a 1.000 gestores. Destes, 138 responderam a pesquisa. Os gestores foram acessados a partir de uma lista disponibilizada por uma empresa de serviços de consultoria e auditoria, a PricewaterhouseCoopers (PwC). 0 questionário foi enviado para os gestores de Recursos Humanos por meio da ferramenta on-line Qualtrics. Utilizouse o questionário eletrônico por conta das vantagens de menores custos, maior velocidade e possibilidade de se distribuir para um grande número de potenciais respondentes (MARTINS; THEÓPHILO, 2009).

O questionário é composto por três partes (Apêndice A). A primeira parte refere-se às variáveis caracterizadoras do respondente, como gênero, idade e grau de escolaridade. A segunda diz respeito às variáveis caracterizadoras da empresa que o respondente trabalha, como seu tamanho, origem, setor em que atua, definição de trabalhador mais velho para o gestor de $\mathrm{RH}$, distribuição etária na organização e idade média dos profissionais nas diferentes posições. A terceira parte do questionário está relacionada às atitudes de gestores de $\mathrm{RH}$ com relação aos trabalhadores mais velhos e a quarta parte do questionário apresenta as práticas de gestão da idade, adotadas ou não pelas empresas. Os dois principais construtos utilizados nesta pesquisa, que contemplam a terceira e quarta parte do questionário, foram Favorabilidade da Atitude (Atitude) e Intensidade de Adoção de Práticas (Práticas). Para medir o grau de concordância com relação aos construtos, utilizou-se uma Escala Likert de 6 pontos. A medida que aumenta o valor na escala, aumenta 0 grau de concordância do respondente com relação ao item.

$\mathrm{O}$ construto Favorabilidade da Atitude representa as percepções de gestores de $\mathrm{RH}$ a respeito dos profissionais com 50 anos ou mais de idade. 0 construto é composto por 38 itens que tiveram como base os estudos abordados no referencial teórico deste trabalho, como os trabalhos Hassel e Perrewe (1995), Taylor e Walker (1998); Ilmarien (2001), Henkens (2005), Dennis e Thomas (2007); Roscigno et al. (2007) e Posthuma e Campion (2009), que apresentaram algumas das percepções de gestores acerca dos profissionais mais velhos. Os itens foram separados em grupos que se referiam a percepções de mesma natureza: postura frente ao trabalho; saúde e desenvolvimento do profissional.

No teste de confiabilidade, o Alpha de Cronbach do construto Favorabilidade da Atitude apresentou valor 0,950 e as estatísticas de escala apresentaram variabilidade de resultados, conforme pode ser visto no Quadro 1.

Quadro 1: Estatística de escala - Atitude

\begin{tabular}{|c|c|c|c|}
\hline Média & Variância & Desvio padrão & N de itens \\
\hline 145,40 & 654,81 & 25,58 & 38 \\
\hline
\end{tabular}

Fonte: Elaborado pelos autores.

Revista Alcance - Eletrônica - vol. 24 - n. 1 - jan./mar. 2017 
O construto Intensidade de Adoção de Práticas representa a adoção de Práticas de Gestão da Idade pelas empresas e é operacionalizado por 27 itens. Estes itens tiveram como base os estudos abordados no referencial teórico deste trabalho, como os trabalhos de Taylor e Walker (1998); Malatest e Associados (2003), Peres (2003); Brooke e Taylor (2005), Naegele e Walker (2006), Armstrong- Stassen (2008) e Lazazzara e Bombelli (2011), que discutiram as práticas de gestão de idade nas organizações. Estes itens foram separados em grupos que se referiam a práticas de mesma natureza: recrutamento e seleção; formação e aprendizagem, saúde e aposentadoria; e, por fim, benefícios. No teste de confiabilidade, o Alpha de Cronbach do construto Intensidade de Adoção de Práticas apresentou valor 0,876 e as estatísticas de escala apresentaram variabilidade de resultados, conforme pode ser visto no Quadro 2.

Quadro 2: Estatísticas de escala - Prática

\begin{tabular}{|c|c|c|c|}
\hline Média & Variância & Desvio padrão & N de itens \\
\hline 72,70 & 364,62 & 19,09 & 27 \\
\hline
\end{tabular}

Fonte: Elaborado pelos autores.

\section{ANÁLISE DOS RESULTADOS}

\subsection{Análise dos Respondentes e das Empresas}

Com relação à participação de homens e mulheres na pesquisa, estas são maioria, somando 74 (54\%) dos respondentes, enquanto os homens somam 64 (46\%) deles. A idade média dos respondentes é de 40 anos e a maioria dos respondentes $(72 \%)$ possuem Pós-Graduação. A maioria das empresas $(70 \%)$ em que os respondentes atuam é de origem brasileira e $30 \%$ são de origem estrangeira. Com relação ao tamanho das empresas, a maioria (42\%) possui receita bruta anual maior do que $\mathrm{R} \$ 300$ milhões. Os setores que apresentaram maior participação na pesquisa são os de prestação de serviços, financeiro e de energia e serviços de utilidade pública. A maior parte dos respondentes, que corresponde a $29 \%$ do total, considera como profissional mais velho aquele com 60 anos ou mais, $23 \%$ definem como profissional mais velho aquele com 55 anos, $18 \%$ como aquele com 50 anos, outros $18 \%$, aquele com 65 anos ou mais de idade e, por fim, os respondentes que o definem como aquele com 45 anos somam $12 \%$.

\subsection{Análise das Percepções de Gestores de RH}

As percepções dos gestores de RH sobre os profissionais com 50 anos ou mais de idade foram investigadas a partir das médias dos itens, que possibilitaram visualizar quais as percepções são positivas ou negativas com relação àqueles profissionais. Para analisar as afirmações, considerou-se a orientação dos itens. Os itens que apresentam média entre 1,0 e 3,50 indicam percepções negativas e aqueles que apresentam média entre 3,51 e 6,0 indicam percepções positivas. O Quadro 3 apresenta as percepções positivas e 0 Quadro 4 apresenta as percepções negativas dos gestores. 
Quadro 3: Percepções positivas

\begin{tabular}{|l|}
\hline \multicolumn{1}{|c|}{ Percepções positivas e médias } \\
\hline Reclamam menos do trabalho - 3,75 \\
\hline Sentem-se mais seguros no emprego - 3,77 \\
\hline Comunicam-se melhor - 3,78 \\
\hline São mais produtivos - 3,81 \\
\hline $\begin{array}{l}\text { Demonstram a mesma capacidade de memorização de informações novas quando comparados aos } \\
\text { profissionais mais jovens - 3,81 }\end{array}$ \\
\hline Trabalham de forma mais árdua - 3,85 \\
\hline Podem aprender novas habilidades tão facilmente quanto outros funcionários - 3,85 \\
\hline Possuem mais facilidade de relacionamento - 3,86 \\
\hline São mais cooperativos - 3,88 \\
\hline Realizam trabalhos com maior qualidade - 3,94 \\
\hline Acompanham o ritmo das atividades de trabalho tanto quanto os funcionários mais jovens - 3,94 \\
\hline Demonstram ter boa condição de saúde para exercer sua função - 4,09 \\
\hline Sofrem acidentes menos graves no trabalho do que os mais jovens - 4,16 \\
\hline São mais organizados - 4,21 \\
\hline São mais confiáveis - 4,23 \\
\hline Possuem maior capacidade de solução de problemas do que os mais jovens - 4,33 \\
\hline São mais obedientes - 4,37 \\
\hline São mais comprometidos - 4,44 \\
\hline Possuem maior conhecimento útil para o dia a dia da empresa - 4,45 \\
\hline Faltam menos - 4,52 \\
\hline Possuem maior capacidade de realizar diagnósticos se comparados aos profissionais mais jovens - 4,63 \\
\hline São mais pontuais - 4,80 \\
\hline São mais fiéis à empresa - 4,83 \\
\hline Possuem maior equilibrio emocional se comparados aos profissionais mais jovens - 4,96 \\
\hline
\end{tabular}

Fonte: Elaborado pelos autores.

Nesta pesquisa, verificou-se que algumas percepções positivas que gestores de RH possuem a respeito dos profissionais mais velhos foram semelhantes aos resultados de estudos internacionais (HASSEL; PERREWE, 1995; HEDGE; BORMAN; LAMMLEIN, 2006; MUNNELL; SASS; SOTO, 2006; ROSCIGNO et al., 2007; DENNIS; THOMAS, 2007; POSTHUMA; CAMPION, 2009). As principais atitudes estão associadas ao maior equilíbrio emocional, à fidelidade à empresa, à pontualidade, à maior capacidade de realizar diagnósticos se comparados aos mais jovens e ao menor absenteísmo. 
Quadro 4: Percepções negativas

\begin{tabular}{|l|}
\hline \multicolumn{1}{|c|}{ Percepções negativas e médias } \\
\hline São capazes de realizar trabalho físico pesado tanto quanto os mais jovens - 2,71 \\
\hline Adaptam-se melhor às mudanças que ocorrem na empresa - 2,92 \\
\hline Preferem atividades de trabalho com mais desafios do que os mais jovens - 3,02 \\
\hline Adaptam-se bem às novas tecnologias introduzidas - 3,04 \\
\hline São mais criativos do que os profissionais mais jovens - 3,08 \\
\hline São mais dispostos a receber treinamento - 3,10 \\
\hline São mais flexíveis no trabalho - 3,17 \\
\hline São mais ágeis no trabalho - 3,26 \\
\hline São menos sujeitos às doenças profissionais do que os mais jovens - 3,26 \\
\hline Mostram-se mais capazes de realizar várias atividades ao mesmo tempo - 3,35 \\
\hline Percebem mais benefícios ao participarem de atividades de aprendizagem do que os mais jovens - 3,37 \\
\hline São tão interessados em aprender novas habilidades quanto os mais jovens - 3,38 \\
\hline São mais proativos - 3,43
\end{tabular}

Fonte: Elaborado pelos autores.

Foram apontadas pelos gestores como percepções negativas as questões relacionadas à realização de trabalho físico pesado, à flexibilidade e à adaptação às mudanças e às novas tecnologias. Essas percepções assemelham-se aos resultados de estudos internacionais sobre o tema, indicando, inclusive, que este é um fator de tensão no ambiente de trabalho (TAYLOR; WALKER, 1998; BROOKE; TAYLOR, 2005; DENNIS; THOMAS, 2007; POSTHUMA; CAMPION, 2009; ROSCIGNO et al., 2007).

Alguns resultados, no entanto, se opõem aos aspectos identificados em estudos internacionais. Embora Maurer (2001) tenha apontado que alguns gestores deixam de submeter profissionais mais velhos a atividades de aprendizagem, esses gestores possuem uma atitude positiva quanto à capacidade de profissionais mais velhos aprenderem novas habilidades tão facilmente quanto outros funcionários. Complementando esse dado, foi possível verificar que, na percepção dos gestores, os profissionais mais velhos não estão dispostos a receber atividades de treinamento. Esta constatação assemelha-se aos resultados obtidos nos estudos de Taylor e Walker (1998), que supõem que isto derive da baixa percepção do profissional mais velho quanto à recompensa que a participação nestas atividades possa gerar. As questões relacionadas à criatividade e ao interesse em aprender novas habilidades, que, para alguns autores (HASSEL; PERREWE, 1995; POSTHUMA; CAMPION, 2009) se mostravam positivas, foram identificadas como atitudes negativas para os gestores respondentes neste trabalho.

Em síntese, pode-se dizer que as percepções de empregadores relativas aos profissionais mais velhos são tanto positivas quanto negativas. Estas últimas podem levar os gestores a adotarem comportamentos discriminatórios no ambiente de trabalho e refletir na ocorrência de ageism no ambiente organizacional (HENKENS, 2005). No entanto, a partir da média das atitudes (3,83), pode-se dizer que os gestores de RH apresentam atitudes relativamente positivas sobre os profissionais mais velhos, pois é superior a 3,51. Mas será que essas atitudes positivas refletem em práticas de gestão da idade? Na subseção seguinte será apresentada a análise das Práticas de Gestão da Idade.

\subsection{Análise das Práticas de Gestão da Idade}

As práticas de gestão da idade foram avaliadas segundo as médias dos itens, que permitem identificar quais práticas de gestão da idade são adotadas na empresa. Para realizar a análise das afirmações, considerouse a orientação dos itens. Para os itens que não estavam orientados em escala inversa, convencionou-se que a 
média entre 1,0 e 3,50 indica a não adoção de práticas e média entre 3,51 e 6,0 indica a adoção de práticas de gestão de idade. No entanto, para as afirmações que indicam escala inversa nos Quadros 5 e 6 , quanto maior 0 grau de concordância, menor a percepção com relação à adoção da prática de gestão de idade pela empresa. Após a coleta de dados, para a análise das afirmações com escala inversa, foi necessário inverter os valores que os gestores atribuíram para o respectivo item, utilizando a fórmula: $R=(H+L)-I$, onde $H$ é o número maior da escala, $L$ é o número mais baixo da escala, I é a resposta a um item e $R$ é 0 item invertido.

Quadro 5: Grau de adoção de práticas elevado

\begin{tabular}{|l|}
\hline \multicolumn{1}{|c|}{ Grau de adoção de práticas elevado e médias } \\
\hline $\begin{array}{l}\text { Em relação a ocupação de cargos de trabalho por profissionais desempregados, é preferível a contratação } \\
\text { dos mais jovens [escala inversa] }-3,53\end{array}$ \\
\hline Adota medidas de promoção de saúde para ter uma força de trabalho mais velha e saudável no futuro - 3,54 \\
\hline $\begin{array}{l}\text { Na seleção para uma vaga, em igualdade de condições, a empresa dá preferência a um profissional mais } \\
\text { jovem [escala inversa] - 3,78 }\end{array}$ \\
$\begin{array}{l}\text { Mediante recrutamento, a empresa seleciona candidatos de diferentes idades para compor os grupos de } \\
\text { trabalho - 3,82 }\end{array}$ \\
\hline A empresa especifica as atividades de trabalho de acordo com a capacidade do profissional - 3,84 \\
\hline $\begin{array}{l}\text { Todos os membros da empresa têm a consciência de que trabalhar com profissionais mais velhos tem a sua } \\
\text { importância - 4,25 }\end{array}$ \\
\hline Na empresa, é recorrente que as atividades de trabalho ocorram entre membros de diferentes idades - 4,44 \\
\hline
\end{tabular}

A partir do Quadro 5, é possível verificar que as principais práticas de gestão da idade que apresentaram algum grau de adoção pelas empresas estão associadas à conscientização dos membros da empresa com relação à importância de se trabalhar com profissionais mais velhos, especificação das atividades de trabalho de acordo com a capacidade do profissional, composição de equipes por membros de diferentes idades e seleção de candidatos de diferentes idades.

Quadro 6: Grau de adoção de práticas reduzido

\begin{tabular}{|l|}
\hline \multicolumn{1}{|c|}{ Grau de adoção de práticas reduzido e médias } \\
\hline $\begin{array}{l}\text { A empresa utiliza letras grandes em materiais de trabalho ou folhetos informativos quando estes são destinados } \\
\text { à força de trabalho mais velha - } 1,64\end{array}$ \\
\hline A empresa possui políticas de remuneração ou benefícios diferenciados para os mais velhos - 1,78 \\
\hline A empresa em que trabalho dispõe de métodos de treinamentos especiais para os mais velhos - 1,80 \\
\hline A empresa oferece planos de carreira diferenciados para os mais velhos - 1,83 \\
\hline A empresa desenvolve campanhas especificas para a seleção de candidatos mais velhos - 1,85 \\
\hline Oferece Plano de Saúde especial aos mais velhos - 1,86 \\
\hline $\begin{array}{l}\text { A empresa oferece preparação para o profissional mais velho se desenvolver em uma atividade autônoma - } \\
2,13\end{array}$ \\
\hline A empresa tem atividades especificas para integração dos profissionais de diferentes gerações - 2,28 \\
\hline $\begin{array}{l}\text { A empresa desenvolve modelos de carreira diferenciados para os mais velhos ou em idade de se aposentarem } \\
\text { (Por exemplo: deixar uma posição gerencial para atuar em projetos e atividades consultivas) - } 2,30\end{array}$ \\
\hline
\end{tabular}

Revista Alcance - Eletrônica - vol. 24 - n. 1 - jan./mar. 2017 


\begin{tabular}{|l|}
\hline A empresa realiza estudos ou relatórios sobre a saúde da força de trabalho envelhecida - 2,30 \\
\hline Conta com um Programa de Preparação para Aposentadoria que envolve os profissionais mais velhos - 2,33 \\
\hline Há possibilidade de profissionais mais velhos realizarem o trabalho de meio período - 2,34 \\
\hline A empresa promove atividades aos mais velhos voltadas especificamente à gestão do conhecimento - 2,38 \\
\hline $\begin{array}{l}\text { A empresa possui postura proativa na contratação de profissionais que já se aposentaram ou que estão } \\
\text { próximos da idade de se aposentar - } 2,50\end{array}$ \\
\hline A empresa tem uma postura proativa de incentivar a contratação de profissionais mais velhos - 2,76 \\
\hline A empresa incentiva a prática de atividade física direcionada aos mais velhos - 2,92 \\
\hline $\begin{array}{l}\text { A empresa incentiva o envolvimento dos profissionais mais velhos em atividades de integração dos mais jovens } \\
\text { à cultura da organização - 3,06 }\end{array}$ \\
\hline $\begin{array}{l}\text { A empresa promove o envolvimento dos mais velhos em atividades de treinamento e desenvolvimento dos mais } \\
\text { jovens - 3,24 }\end{array}$ \\
\hline Na área da saúde, a empresa oferece check-up anual para os trabalhadores mais velhos - 3,26 \\
\hline Na empresa, os mais velhos realizam atividades de mentoring para os mais jovens - 3,31 \\
\hline
\end{tabular}

Fonte: Elaborado pelos autores.

Apesar de algumas práticas de gestão da idade terem sido identificadas, o Construto Prática apresentou média 2,78, indicando que, de forma geral, para os gestores de $\mathrm{RH}$ respondentes da pesquisa, as práticas de gestão da idade não são adotadas pelas empresas. Este resultado confirma os achados de outros estudos internacionais (ARMSTRONG-STASSEN, 2008; ZNIDARSIC; DIMOVKSKI, 2009), de que as organizações estão envolvidas com estas práticas de maneira bastante limitada e que a idade ainda se mostra um fator limitante para que o profissional mais velho se insira ou se mantenha no mercado de trabalho (CARVALHO, 2009).

\subsection{Relação entre Percepções de gestores e Práticas de Gestão da Idade}

Para averiguar a relação entre os construtos Favorabilidade da Atitude (Atitude) e Intensidade de Adoção de Práticas (Práticas), foi calculado o Coeficiente de Correlação de Pearson, o qual apresentou valor 0,282, o que indica que as variáveis estão fracamente correlacionadas. A Figura 1 apresenta a dispersão dos dados.

Figura 1: Gráfico de dispersão

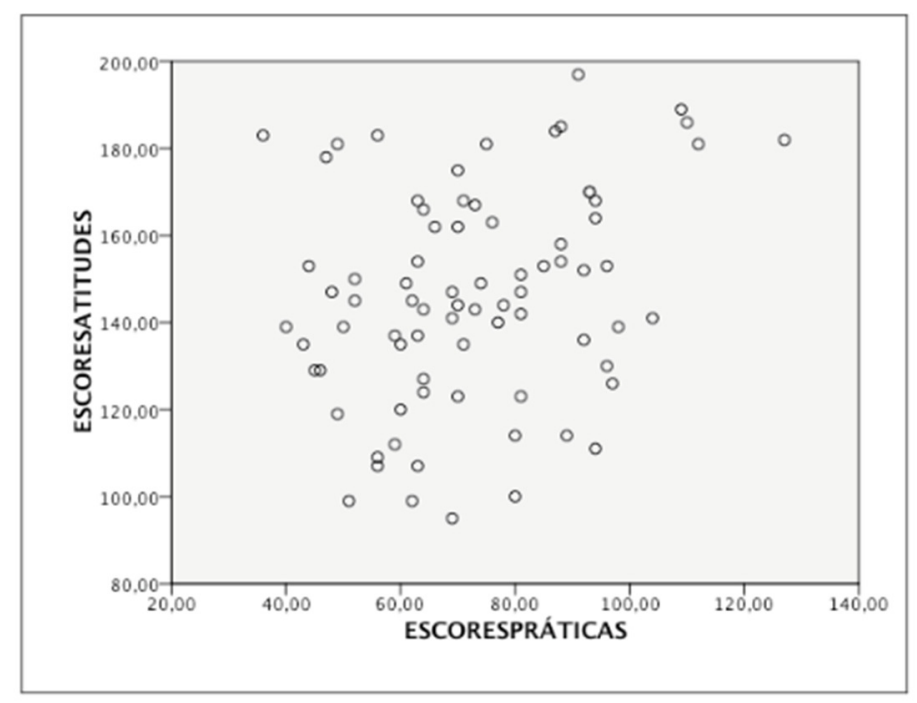

Fonte: Elaborado pelos autores. 
Foi possível averiguar que a correlação entre as atitudes de gestores de $\mathrm{RH}$ sobre os profissionais mais velhos e a adoção de práticas de gestão da idade pelas empresas é fraca. Assim sendo, as atitudes positivas dos gestores de RH respondentes desta pesquisa não implicaram práticas efetivas de gestão da idade nas empresas. A verificação do baixo grau de adoção de práticas de gestão da idade leva à reflexão sobre a existência, no âmbito organizacional, do ageism institucional, o qual se refere às regras, às missões e às práticas estabelecidas que discriminam as pessoas mais velhas ou grupos com base na idade (DENNIS; THOMAS, 2007). Para Goldani (2010), o ageism é um desafio que a sociedade deve enfrentar e eliminar mediante a conscientização e a educação, como também mediante intervenções políticas. Entretanto, segundo Fineman (2011), somente organizações atentas irão desenvolver práticas de $\mathrm{RH}$ que forneçam oportunidades para que os recursos provenientes dos profissionais mais velhos sejam aproveitados e valorizados.

\section{CONSIDERAÇÕES FINAIS}

Atualmente, o país enfrenta um processo de transição demográfica marcada por três fatores: o primeiro diz respeito às baixas taxas de fecundidade; o segundo, às baixas taxas de mortalidade e, por último, o aumento da expectativa de vida do brasileiro. A consequência destes fatores é o envelhecimento populacional. Projeções indicam que, a partir de meados de 2020 , se iniciará no Brasil um processo de redução da força de trabalho e, a partir de 2040, muitos dos ocupados serão profissionais com mais de 45 anos, que serão responsáveis por, aproximadamente, $56,3 \%$ da futura População em Idade Ativa.

Por esta razão, é relevante compreender como o envelhecimento dos profissionais tem sido compreendido nas organizações, ou seja, quais as percepções acerca do profissional mais velho e quais as práticas de gestão da idade têm sido implementadas. Então, com o intuito de responder às perguntas: quais são as percepções positivas e negativas de gestores de $\mathrm{RH}$ a respeito dos profissionais mais velhos e quais são as práticas de gestão da idade adotadas pelas empresas pesquisadas?, a pesquisa com 138 gestores de RH de empresas que atuam no Brasil possibilitou verificar como o envelhecimento do profissional está sendo tratado nas empresas pesquisadas.

A pesquisa mostrou que as percepções sobre profissionais mais velhos são diversificadas e que, de forma geral, elas são muito semelhantes às identificadas em estudos internacionais sobre o tema. Entretanto, apesar de as percepções serem relativamente positivas, as empresas em que os gestores atuam não apresentam práticas de gestão de idade que visam eliminar as barreiras etárias e promover a diversidade no ambiente organizacional.

As principais práticas de gestão da idade que apresentaram algum grau de adoção pelas empresas estão associadas à conscientização dos membros da empresa com relação à importância de se trabalhar com profissionais mais velhos, especificação das atividades de trabalho de acordo com a capacidade do profissional, composição de equipes por membros de diferentes idades e seleção de candidatos de diferentes idades.

As principais práticas que não são adotadas, na visão dos gestores, estão relacionadas às práticas de ergonomia e saúde que objetivam minimizar os efeitos do envelhecimento, políticas de remuneração ou benefícios diferenciados aos mais velhos, métodos de treinamento e campanhas especiais para a seleção de candidatos mais velhos, oferta de planos de carreira diferenciados e oferta de preparação para desenvolvimento de uma atividade autônoma. Além disso, apesar dos gestores acreditarem que os mais velhos apresentem características como produtividade, confiabilidade, comprometimento e pontualidade, entre outros, notou-se que, na maior parte das vezes, as empresas não adotaram uma postura proativa na contratação destes profissionais ou que estão próximos da idade de se aposentar.

Portanto, os resultados obtidos nesta pesquisa permitiram identificar que, não obstante a população brasileira esteja envelhecendo de forma acelerada, caminhando para o futuro de uma força de trabalho envelhecida, as empresas se mostram despreparadas para enfrentar este cenário. Além disso, foi possível verificar que 0 ageism institucional se faz presente na realidade de trabalho brasileira. Este trabalho alerta para a necessidade da continuidade da pesquisa sobre o tema na área de estudos organizacionais e a sensibilização de gestores a direcionarem sua atenção para o cuidado e valorização do profissional com mais idade, por meio de medidas que visem protegê-los durante sua atuação nos próximos anos.

\section{REFERÊNCIAS}

ANGELONI, S.; BORGONOVI, E. An ageing world and the challenges for a model of sustainable social change.

Revista Alcance - Eletrônica - vol. 24 - n. 1 - jan./mar. 2017 
Journal of Management Development, v. 35, 2016.

ARMSTRONG-STASSEN, M. Human Resource practices for mature workers and why aren't employers using them? Asia Pacific Journal of Human Resources, 2008.

BANCO MUNDIAL. Envelhecendo em um Brasil mais velho, 2011.

BRASIL. Constituição da República Federativa do Brasil. 1998. Recuperado de:

$<$ http://www.planalto.gov.br/ccivil_03/constituicao/constituicao.htm>.

BROOKE, L.; TAYLOR, P. Older workers and employment: managing age relations. Ageing and Society, v. 25, n. 3, p. 415-29, 2005.

BUTLER, R. Dispelling ageism: the cross-cutting intervention. Annals of the American Academy of Political and Social Science, p. 138-47, 1989.

BUTLER, R. The longevity revolution: the benefits and challenge of living a longlife. PublicAffairs. 2010.

CARVALHO, A. S. Gestão de pessoas e envelhecimento: sentido do trabalho para o idoso. São Paulo. In: Encontro da ANPAD, São Paulo, SP, Brasil, 33. Anais... Rio de Janeiro: ANPAD, 2009.

CLAES, R.; HEYMAND, M. HR professionals' views on work motivation and retention of older workers: a focus group study. Career Development International, v. 13, n. 2, p. 95-111, 2008.

DENNIS, H.; THOMAS, K. Ageism in the workplace. São Paulo: Spring, 2007.

FONTOURA, D. DOS S.; PICCININI, V. C. Envelhecimento populacional e gestão de pessoas: pesquisas internacionais e notas para o Brasil. In: Encontro da ANPAD, Rio de Janeiro, RJ, Brasil, 36. Anais... Rio de Janeiro: ANPAD, 2012.

FINEMAN, S. Organizing age. Oxford: Oxford. 2011.

FRANÇA, L. H. de F. P. O envelhecimento populacional e seu reflexo nas organizações: a importância da educação ao longo da vida. Boletim Técnico do Senac, Rio de Janeiro, v. 37, n. 2, 2011.

FUNDO DE POPULAÇÃO DAS NAÇÕES UNIDAS. Envelhecimento no Século XXI: Celebração e desafio. Nova York. 2012.

GOLDANI, A. M. Desafios do preconceito etário no Brasil. Educação \& Sociedade, Campinas, v. 31, n. 111, 2010.

GAVRILOV, L. A; HEUVELINE, P. Aging of population. In: DEMENY, P.; MCNICOLL, G. The Encyclopedia of Population, New York, Macmillan Reference, USA, 2003.

HASSEL, B. L.; PERREWE, P. L. An examination of beliefs about older workers: do stereotypes still exist? Journal of Organizational Behavior, v. 16, p. 457-468, 1995.

HEDGE, J. M.; BORMAN, W.C.; LAMMLEIN, S.E. The aging workforce: Realities, myths, and implications for organizations. Washington DC: American Psychological Association, 2006.

HENNEKAM, S. Career success of older workers: the influence of social skills and continuous learning ability. Journal of Management Development, v. 34, n. 9, p. 1113 -1133, 2015.

HENNEKAM, S.; HERRBACH, O. The influence of age-awareness versus general HRM practices on the retirement decision of older workers. Personnel Review, v. 44, n. 1, p. 3-21, 2015.

HENKENS, K. Stereotyping older workers and retirement: the managers' point of view. Canadian Journal on Aging, v. 24, n. 4, p. 353-366, 2005.

HENRARD, J. C. Cultural problems of ageing especially regarding gender and intergenerational equity. Social Science and Medicine, v. 43, n. 5, p. 667-80, 1996.

INSTITUTO BRASILEIRO DE GEOGRAFIA E ESTATÍSTICA (IBGE). Síntese de Indicadores Sociais: uma análise das condições de vida da população brasileira. Rio de Janeiro, 2013.

KOOIJ, D. et al. Older workers' motivation to continue work: five meanings of age. A conceptual review. Journal of Managerial Psychology, n. 23, p. 364-394, 2008. 
LAZAZZARA, A.; BOMBELLI, M. C. HRM practices for an ageing italian workforce: the role of training. Journal of European Industrial Training, v. 35, n. 8, 2011.

LOCATELLI, P. A. P. C.; FONTOURA, D. dos S. Envelhecimento Populacional e os Estudos Em Administração. Gestão e Sociedade, Belo Horizonte, v. 7, n. 17, p. 273-300, 2013.

LORETTO, W.; WHITE, P. Employers' attitudes, practices and policies toward older workers. Human Resource Management Journal, v. 16, n. 3, p. 313-330, 2006.

LOTH, G. B.; SILVEIRA, NEREIDA. Etarismo nas organizações: um estudo dos estereótipos em trabalhadores envelhecentes. Revista de Ciências da Administração, v. 16, n. 19, p. 65-82, 2014.

MALATEST, R. A.; ASSOCIADOS LTDA. The aging workforce and human resources development implications for sector councils. Ottawa, Canadá. 2003.

MANPOWER. The new agenda for an older workforce. Milwaukee, WI: Manpower Inc. 2007.

MARTINS, G. de A.; THEÓPHILO, C. R. Metodologia da investigação científica para ciências sociais aplicadas. 2. ed. São Paulo: Atlas, 2009.

MUNNELL, A. H.; SASS, S. A.; SOTO, M. Employer attitudes towards older workers: survey results. Work Opportunities for Older Americans, v. 3, 2006.

NAEGELE, G.; WALKER, A. A guide to good practices in age management. European foundation for the improvement of living and working conditions. 2006.

PALMORE, E. B. Ageism: negative and positive. 2. ed. New York: Springer. 1999.

PERES, M. A. de C. As estratégias empresariais e a exclusão por idade. Revista Gerenciais, São Paulo, v. 2, p. 15-21, 2003.

PHILLIPS, D. R.; SIU, O.-L. Global Aging and Aging Workers. The Oxford. Handbook of Work and Aging. 2012.

POSTHUMA, R. A.; CAMPION, M. A. Age stereotypes in the workplace: common stereotypes, moderators, and future research directions? Journal of Management, v. 35, n. 1, 2009.

ROSCIGNO, V. J. et al. Age discrimination, social closure and employment. Social Forces, v. 86, n. 1, 2007.

SILVA, R. C.; DUTRA, J. S.; VELOSO, E. F. R.; TREVISAN, L. N. As gerações em distintos contextos organizacionais. Gestão e Regionalidade, v. 30, n. 89, 2014.

TAYLOR, P.; WALKER, A. Employers and older workers: attitudes and employment practices. Ageing \& Society, Cambridge University Press, v. 18, n. 6, p. 641-58, 1998.

VASCONCELOS, A. F. Older workers: some critical societal and organizational challenges. Journal of Management Development, v. 34, p. 352-372, 2015.

ZNIDARSIC, J.; DIMOVSKI, V. Age management: a new paradigm. In: HRM Within Slovenian Enterprises. The Journal of Applied Business Research, v. 25, n. 3, 2009. 


\section{APÊNDICE A - QUESTIONÁRIO DA PESQUISA}

\section{Nosso objetivo}

Nossa pesquisa visa identificar as principais percepções dos gestores de Recursos Humanos em relação às diferentes características das gerações que compõem 0 ambiente de trabalho e quais têm sido as práticas adotadas pela organização sobre esse tema.

\section{A. CARACTERIZAÇÃO DO RESPONDENTE}

1 - Sexo

a) $\quad M$

b) $F$

2 - Idade:

3 - Qual o seu grau de escolaridade?

a) Ensino Fundamental

b) Ensino Médio

c) Ensino Superior

d) Pós-Graduação

\section{B. CARACTERIZAÇÃO DA EMPRESA}

4 - A empresa que você trabalha é de origem:

a) Brasileira

b) Estrangeira

5 - Setor em que a empresa atua:

a) Agronegócios

b) Automotivo

c) Celulose, papel e embalagens

d) Produtos de consumo e varejo

e) Energia e serviços de utilidade pública

f) Engenharia e construção

g) Tecnologia e informação

h) Comunicação, Telecom e entretenimento

i) Saúde

j) Financeiro

k) Siderurgia e metalurgia

l) Mineração

m) Óleo e gás

n) Químico e petroquímico

o) Serviços

p) Outro:

6 - Receita bruta anual da empresa (no Brasil):

a) Menor ou igual a $\mathrm{R} \$ 16$ milhões

b) Maior que $R \$ 16$ milhões e menor ou igual a $R \$ 90$ milhões

c) Maior que $\mathrm{R} \$ 90$ milhões e menor ou igual a $\mathrm{R} \$ 300$ milhões

d) Maior que $\mathrm{R} \$ 300$ milhões

7 - Que idade caracterizaria para você um profissional como mais velho?

a) Acima de 45

b) Acima de 50

c) Acima de 55 
d) Acima de 60

e) Acima de 65

8 - Quantos profissionais são encontrados na empresa nas seguintes categorias:

\begin{tabular}{|l|l|l|l|}
\hline Faixa Etária & Homem & Mulher & Total \\
\hline $18-29$ & & & \\
\hline $30-44 \mathrm{~s}$ & & & \\
\hline $45-64 \mathrm{~s}$ & & & \\
\hline 65 ou mais & & & \\
\hline
\end{tabular}

9 - Qual a idade média dos profissionais que se enquadram nas seguintes posições:

\begin{tabular}{|l|l|}
\hline Posição & Idade Média \\
\hline Diretores/executivos & \\
\hline Gerentes & \\
\hline Supervisores/coordenadores & \\
\hline Analistas e equipe operacional & \\
\hline
\end{tabular}

\section{PERCEPÇÕES SOBRE TRABALHADORES MAIS VELHOS}

As afirmações a seguir referem-se à sua percepção com relação aos trabalhadores mais velhos na empresa em que você trabalha. Atribua uma nota de 0 a 6 para cada afirmação. Na medida em que o número aumenta na escala, aumenta o seu grau de concordância. Considere como trabalhador mais velho aquele com $\mathbf{5 0}$ anos ou mais.

\section{POSTURA FRENTE AO TRABALHO}

Com relação à postura frente ao trabalho, se comparado aos profissionais mais jovens, os profissionais mais velhos:

\section{Discordo Totalmente}

1

2

3

4

5

10 - Trabalham de forma mais árdua

11 - São mais pontuais no trabalho

12 - São mais confiáveis

13 - Realizam trabalhos com maior qualidade

14 - Sentem-se mais seguros no emprego

15 - São mais proativos

16 - Faltam menos

17 - São mais ágeis

18 - São mais comprometidos no trabalho

19 - São mais fiéis à empresa

20 - São mais flexíveis

21 - São mais cooperativos

22 - São mais produtivos

23 - São mais organizados

24 - São mais obedientes

25 - Reclamam menos

26 - Adaptam-se melhor às mudanças que ocorrem na empresa

27 - Possuem mais conhecimento útil para o dia a dia da empresa

28 - Mostram-se mais capazes de realizar várias atividades ao mesmo tempo

29 - Comunicam-se melhor

30 - Possuem maior facilidade de relacionamento

Revista Alcance - Eletrônica - vol. 24 - n. 1 - jan./mar. 2017 


\section{SAÚDE}

Em relação ao estado de saúde, os profissionais mais velhos:

Discordo Totalmente 1
3

\section{Concordo Totalmente 6}

31 - Demonstram ter boa condição de saúde para exercer sua função

32 - Sofrem menos acidentes no trabalho do que os mais jovens

33 - Sofrem acidentes menos graves no trabalho do que os mais jovens

34 - São menos sujeitos a doenças profissionais do que os mais jovens

35 - São capazes de realizar trabalho físico pesado tanto quanto os mais jovens

\section{DESENVOLVIMENTO DO PROFISSIONAL}

Em relação ao desenvolvimento do profissional, os mais velhos:

\section{Discordo Totalmente \\ 1}

2
3

4

5

\section{Concordo Totalmente 6}

36 - São mais dispostos a receber os treinamentos proporcionados pela empresa do que os mais jovens

37 - São tão interessados em aprender novas habilidades quanto os mais jovens

38 - Adaptam-se bem às novas tecnologias introduzidas

39 - Percebem mais benefícios ao participarem de atividades de aprendizagem do que os mais jovens

40 - Preferem atividades de trabalho com mais desafios do que os mais jovens

41 - São mais criativos do que os mais jovens

42 - Possuem maior capacidade de solução de problemas do que os mais jovens

43 - Acompanham o ritmo das atividades de trabalho tanto quanto os funcionários mais jovens

44 - Demonstram a mesma capacidade de memorização de informações novas quando comparados aos mais jovens

45 - Possuem maior equilíbrio emocional se comparado aos mais jovens

46 - Podem aprender novas habilidades tão facilmente quanto outros funcionários

47 - Possuem maior capacidade de realizar diagnósticos se comparado aos profissionais mais jovens.

\section{PRÁTICAS DE GESTÃO DA EMPRESA}

As afirmações a seguir referem-se à sua percepção com relação às práticas de gestão adotadas pela empresa que você trabalha. Atribua uma nota de 0 a 6 para cada afirmação. Na medida em que o número aumenta na escala, aumenta o seu grau de concordância. Considere como trabalhador mais velho aquele com 50 anos ou mais.

\section{Recrutamento e Seleção}

Sobre as práticas de recrutamento e seleção da empresa em que trabalho:

Discordo Totalmente

$$
1
$$

\section{2}

3
4

\section{Concordo Totalmente}

5

48 - A empresa especifica as atividades de trabalho de acordo com a capacidade do profissional

49 - Todos os membros da empresa têm a consciência de que trabalhar com os mais velhos tem a sua importância

50 - Em relação à ocupação de cargos de trabalho por profissionais desempregados, é preferivel a contratação dos mais jovens.

51 - A empresa desenvolve campanhas específicas para a seleção de candidatos mais velhos 
52 - A empresa possui postura proativa na contratação de profissionais que já se aposentaram ou que estão próximos da idade de se aposentar

53 - Na seleção para uma vaga, em igualdade de condições, a empresa dá preferência a um profissional mais jovem

54 - Por meio de recrutamento, a empresa seleciona candidatos de diferentes idades para compor os grupos de trabalho

55 - A empresa tem uma postura proativa de incentivar a contratação de profissionais mais velhos

\section{Formação e Aprendizagem}

Sobre as práticas de formação e aprendizagem da empresa em que trabalho:

\section{Discordo Totalmente}

1
2
4

\section{Concordo Totalmente}

6

56 - Na empresa, os mais velhos realizam atividades de mentoring para os mais jovens

57 - A empresa tem atividades específicas para integração dos profissionais de diferentes gerações

58 - A empresa em que trabalho dispõe de métodos de treinamento especiais para os mais velhos

59 - Na empresa, é recorrente que as atividades de trabalho ocorram entre membros de diferentes idades

60 - A empresa desenvolve modelos de carreira diferenciados para os mais velhos ou em idade de aposentar (por exemplo: deixar uma posição gerencial para atuar em projetos e atividades consultivas)

61 - A empresa promove atividades aos mais velhos voltadas especificamente à gestão do conhecimento

62 - A empresa incentiva o envolvimento dos mais velhos em atividades de integração dos mais jovens à cultura da organização

63 - A empresa promove o envolvimento dos mais velhos em atividades de treinamento e desenvolvimento dos mais jovens

64 - A empresa oferece preparação para o profissional mais velho se desenvolver em uma atividade autônoma

\section{Saúde e Aposentadoria}

Em relação às práticas de saúde e aposentadoria, a empresa em que trabalho:

\section{Discordo Totalmente}

1
2
4

\section{Concordo Totalmente}

5

65 - Conta com um Programa de Preparação para Aposentadoria que envolve os profissionais mais velhos 66 - Adota medidas de promoção da saúde para ter uma força de trabalho mais velha e saudável no futuro 67 - A empresa oferece planos de carreira diferenciados para profissionais mais velhos.

68 - Oferece Plano de Saúde especial aos mais velhos

69 - A empresa adota como medida de ergonomia adaptada para a força de trabalho mais velha letras grandes em materiais de trabalho ou folhetos informativos

70 - $\mathrm{Na}$ área da saúde, a empresa oferece checkup anual para os mais velhos

71 - A empresa incentiva a prática de atividade física direcionada aos mais velhos

72 - A empresa realiza estudos ou relatórios sobre saúde da força de trabalho

\section{Benefícios}

Em relação aos benefícios, na empresa em que trabalho:

Discordo Totalmente

1
2

3
4

\section{Concordo Totalmente}

6

73 - Há possibilidade de profissionais mais velhos realizarem o trabalho de meio período

74 - A empresa possui políticas de remuneração ou benefício diferenciados para os mais velhos

Revista Alcance - Eletrônica - vol. 24 - n. 1 - jan./mar. 2017 
75 - Quais os principais benefícios enxergados pela empresa em ter profissionais mais velhos compondo as equipes de trabalho? Assinale no máximo cinco opções

a) Capacidade de disseminar os comportamentos esperados pela organização, dos mais velhos para os mais novos

b) Oportunidade de estabelecimento de programas de mentoring dos mais velhos para os mais novos

c) Diversidade de ideias e pontos de vistas dentro das equipes, enriquecendo abordagens de trabalho ou formas de resolução de problemas

d) Capacidade de disseminar a cultura da organização para os mais novos

e) Experiência profissional com relação aos conhecimentos técnicos adquiridos ao longo da carreira

f) É uma opção aos empregadores para a carência de profissionais qualificados no mercado de trabalho local

g) Facilidade em lidar com pessoas e trabalhar em equipe

h) Boa repercussão da imagem da empresa em virtude da inserção do mais velho

i) Comprometimento e senso de responsabilidade demonstrados pelos profissionais mais velhos

j) Capacidade de solucionar problemas com facilidade

76 - Quais as principais barreiras enxergadas pela empresa em ter profissionais mais velhos compondo as equipes de trabalho? Assinale no máximo cinco opções

a) Conflitos de ideias e perspectivas nas equipes de trabalho, comprometendo o clima da equipe

b) Dificuldades de reconhecimento da liderança quando os mais velhos são liderados pelos mais novos

c) Os mais velhos podem comprometer o desenvolvimento de jovens profissionais

d) Acomodação por conta da expectativa da aposentadoria

e) Dificuldade em se manter atualizado com relação às novas exigências do mercado

f) Exigência de maiores salários

g) Dificuldade em aceitar os jovens como futuros talentos da empresa

h) Dificuldade em lidar com as novas tecnologias

i) Limitações físicas e mentais do profissional mais velho

j) Falta de flexibilidade e adaptação às mudanças ocorridas na empresa 